\title{
Interactive Therapeutic Multi-sensory Environment for Cerebral Palsy People
}

\author{
Cesar Mauri ${ }^{1}$, Agusti Solanas ${ }^{2}$, Toni Granollers ${ }^{1}$, Joan Bagés ${ }^{3}$, \\ and Mabel García ${ }^{4}$ \\ ${ }^{1}$ GRIHO, HCI research group (University of Lleida) \\ ${ }^{2}$ CRISES Research Group (Rovira i Virgili University) \\ ${ }^{3}$ Centre de Reserche Informatique et Creation Musicale (University of Paris) \\ ${ }^{4}$ Associació Provincial de Paràlisi Cerebral, Tarragona \\ cesar.mauri@urv.cat, agusti.solanas@urv.cat, tonig@diei.udl.cat, \\ joanbir@hotmail.com, mggs@tinet.org
}

\begin{abstract}
The Interactive Therapeutic Sensory Environment (ITSE) research project offers new opportunities on stimulation, interaction and interactive creation for people with moderate and severe mental and physical disabilities. Mainly based on computer vision techniques, the ITSE project allows the gathering of users' gestures and their transformation into images, sounds and vibrations. Currently, in the APPC ${ }^{1}$, we are working in a prototype that is capable of generating sounds based on the users' motion and to process digitally the vocal sounds of the users. Tests with impaired users show that ITSE promotes participation, engagement and play. In this paper, we briefly describe the ITSE system, the experimental methodology, the preliminary results and some future goals.
\end{abstract}

Keywords: Cerebral Palsy, Elderly, Disabled People, Artificial Vision.

\section{Introduction and Motivation}

This article presents the Interactive Therapeutic Sensory Environment (ITSE) project. Its main aim is to investigate, develop and apply a system able to analyze the user's gestures and vocal sounds (by means of web cameras and microphones), and to transform them into images, sounds and vibration. By doing so, the system creates a sensitive space with a multimodal feedback highly coupled with the input.

The project was born in the APPC and the reduced number of users that were able to interact with computers was the main motivation. Although several assistive technology gadgets and special software applications were used, most users "simply" could not understand the interaction procedures. We believed that a high interactive activity (reinforced with sounds and images closely related with gestures) would be more easily accessible to most users despite their sensory, motor and cognitive impairments.

${ }^{1}$ APPC (in Catalan stands for Associació Provincial de Paràlisi Cerebral), Cerebral Palsy Centre of Tarragona. APPC is located in Catalonia (Spain). 
Our goal is to turn the system into a tool that has plenty of activities allowing users to explore, express, play and enjoy. Moreover, by means of these activities, users may develop capabilities -sometimes unconsciously- such as participation, communication or creativity and, consequently, they improve their quality of live and wellbeing. The project focuses on people with cerebral palsy but it could also benefit people with other cognitive disabilities.

\section{State of the Art}

In spite of the importance of studying the interaction capabilities of cerebral palsy people, the number of related references in the literature is quite limited. Next three main lines/projects constitute the most relevant advances up to now:

1. P. Ellis and Soundbeam. In the early 90s, P. Ellis started to use the so-called sound therapy with Soundbeam ${ }^{2}$ on children with autism and profound and multiple learning disabilities. Sound therapy combines the power of a contactless interactive space with an aesthetic response to sound. This encourages users' interaction and communicative skills development 4. In 1998, Ellis introduced the VibroAcoustic Sound Therapy that included a microphone and a vibroacoustic device (VAD) to the system. Since 2004 Ellis, from the iMUSE laboratory, focuses on the elderly and studies the addition of image feedback to the system 5.

2. T. Brooks and Soundscapes. Soundscapes 1 refers to a body function capture library and to a collection of software able to generate a response from the gesture. One of the uses of Soundscapes is as an "expression amplifier" for people with disabilities. It allows users to generate images and sounds from body motion. The main idea is to create a virtual interactive space (VIS) where users like to be and where motivation and creativity are promoted through play and fun. Several European projects (e.g. 2) show the therapeutic use of Soundscapes.

3. The Mediate project 8 proposed a multi-sensory installation whose main goal is to let children with autism to have fun, play, explore and create in a controlled and secure environment. The project carried out psychological studies and provided parents with an environment where see their children playing.

In addition to these three main lines, other projects can be found in the literature, namely Intellivision 3 and Virtual Music Instrument (VMI) 6.

\section{The ITSE Project}

Promoting the participation of people with severe mental disabilities -that normally remain passive- would lead to a series of reactions that would foster their development. These people are usually withdrawn and isolated from their surroundings due to their limited communication abilities. Providing them with a channel that let them communicate, express feelings, and even create, will contribute to the improvement of their quality of life. To cope with this task it is necessary to have the right tools that

\footnotetext{
${ }^{2}$ Soundbeam is a device that uses an ultrasound sensor to capture user's motion and translates it into sounds (in form of MIDI notes).
} 
provide multimodal interaction in an efficient, simple, and cheap way. Based on our previous experiences 7 , we believe that computer vision techniques can play an important role due to their versatility. The ITSE system consists of two software modules: (a) The motion capture, an application that captures and processes a video stream in real time. It extracts several parameters such as the direction and quantity of movement, the position of a color marker and the index of global activity. And, (b) The audiovisual generation, an application developed using Max/MSP. It processes the information from the capture module and generates images and sounds. MIDI protocol is used for the communication between both modules. Its main features are: (i) a real-time digital sound effect processor (including effects like reverb, chorus, pitch change and echo) that allows users to play with their voice, (ii) a motion-based virtual instrument that allows users to play MIDI instruments by means of their gestures, and (iii) a sound visualizer (using an external application called R $4^{3}$ ). In order to test the ITSE system we have equipped, in the APPC facilities, a quiet room with a computer, a sound system, a large screen, a web camera and a camcorder. Also we have selected several participants for the experiments. Currently, we distinguish among three groups of people according to their cognitive abilities: (i) people able to understand the procedure of the activities, (ii) people that initially do not understand the procedure of the activities but can understand it after some training and, (iii) severe cognitively impaired people that cannot understand the activities. Usually three people are present in the experiments: the researcher (managing the system and taking annotations), the facilitator (a professional close to the user, typically a teacher or therapist) and the user.

Experimentation sessions (between 15 and $30 \mathrm{~min}$.) start welcoming the user and suggesting him an activity (essentially playing with his voice or playing a virtual instrument). This suggestion and the specific arrangements of the activity (level of complexity/difficulty and other parameters) depend on the abilities of the user and his success rate observed in previous sessions. The facilitator starts encouraging the user to interact, but without forcing him. In fact, we try to intervene as little as possible. Depending on the user behavior during the session, the researcher can smoothly change the parameters of the activity or switch to another one. The session ends when the assigned time span expires or when the user becomes tired. The whole session is recorded in video including the final comments made by the researcher and the facilitator.

The analyses of video recordings and annotations include studying several variables such as the type of attention and span, satisfaction, inhibition, etc. After a year of experimentation, we can conclude that the ITSE project promotes attention, perception, communicative intention, muscular control, expression, "connection" with the surrounding environment, imagination, participation, play and funny engagement. For instance, for some people we are specifically interested in seeing if action-reaction relationship is well understood. To prove that, we switch off audio and video for a while and look for user's behavior changes, like looking at the facilitator or changing the facial expression. It allowed us to demonstrate that some users are able to understand the action-reaction relationship. Other results include the presence of previously unseen behavior or physical movements. However, the studies are still in a preliminary stage. A demonstration video is available in 9 .

${ }^{3}$ http://www.rabidhamster.org/R4/ 


\section{Conclusions and Future Work}

Although the idea of a contactless interactive multi-sensory environment is not new, our proposal tries to make such systems wide available reducing costs and simplifying the setups. This is a challenging project due to the severe impairments of the users and the interdisciplinarity of the task. However, after one year of experiments, results are very promising. Future improvements include to add a learning/adaptive module, to better characterize user's profiles by means of ontologies, to deploy a detailed analysis methodology, to improve usability to avoid the need for technical experts, and to develop a software framework in which new activities and sensors could be easily added.

\section{References}

1. Brooks, T.: Virtual interactive space (VIS) as a movement capture interface tool giving multimedia. In: Intl. Congr. World Confed Physical Therapy, Japan (1999)

2. Brooks, T.: CAREHERE. In: Intl. Conf. Disability, Virtual Reality \& Assoc. Tech., Oxford, England, pp. 191-198 (2004)

3. Brumback, C., Borrisov, L., Galusha, J., DiIorio, A.: Intellivision. In: The 6th Intl. Conf. Ubiquitous Computing, UK (2004)

4. Ellis, P.: Incidental music; a case study in the development of sound therapy. The British Journal of Music Education 12, 59-70 (1995)

5. Ellis, P.: The development of interactive multisensory environments for expression. Keynote to Luxembourg Society for Music Therapy (2006)

6. Lamont, A., Knox, R., Chau, T., et al.: Converting movements to music new musical exploration opportunities for children in rehabilitation. In: Canadian Asso. for Music Therapy $29^{\text {th }}$ Annual Conference, Regina, Canada, pp. 26-30 (2002)

7. Mauri, C., Granollers, T., Lores, J., García, M.: Computer vision interaction for people with severe movement restrictions. Human Tech. 2(1), 38-54 (2006)

8. Pares, N., Carreras, A., Durany, J., Ferrer, J., Freixa, P., Gomez, D., Kruglanski, O., Pares, R., Ribas, I.J., Soler, M., Sanjurjo, A.: Promotion of creative activity in children with severe autism through visuals in an interactive multisensory environment. In: Proc. of the 2005 conference on Interaction design and children, pp. 110-116. ACM, Boulder (2005)

9. http://video.google.es/videoplay?docid=-6074387707245897409 